\title{
INTEGRABLE LONG-RANGE CORRELATIONS AND GENERALIZED STATISTICS
}

\author{
ANDREAS OSTERLOH ${ }^{1,2}$, LUIGI AMICO ${ }^{1}$, AND ULRICH ECKERN ${ }^{2}$ \\ ${ }^{1}$ DMFCI, Universìtá di Catania, Viale A. Doria 6, 95125 Catania, Italy \\ Istituto Nazionale per la Fisica della Materia (INFM), Unitá di Catania \\ ${ }^{2}$ Institut für Physik, Universität Augsburg, 86135 Augsbürg, Germany
}

We define one-dimensional generalized statistics based on second quantization. Particles with this kind of statistics are higher-dimensional representations of the symmetric group. They are realizable in terms of fermionic creation and annihilation operators iff the deformation is uncorrelated. Inserting this realization into the Hubbard model for particles with such a statistics, a fermionic model with long-range correlations is created. Extending a work by $\mathbf{H}$. Schula and B. S. Shastry ${ }^{7}$ we find conditions for the long-range correlations and hence for the statistics parameters in order that the model is solvable.

\section{Introduction}

The statistics of degrees of freedom drastically affects the physical properties of a many-particle system. Besides bosonic and fermionic statistics, a continuous family of intermediate statistics serves to explain important effects in two- or one-dimensional physics. Here we focus on the peculiar case of one dimension. ${ }^{1}$ We define Deformed Exchange Statistics (DES) as a specific deformation of fermionic commutation rules. ${ }^{2}$ It has been applied to investigate how robust is the solvability by Coordinate Bethe Ansatz (CBA) ${ }^{3}$ of the XXZ and the Hubbard model (HM) with respect to such a modification of the particle content. This direct approach has the disadvantage to be based on trial and error. Furthermore from numerical diagonalization for small systems we found that many DES models, though not directly accessible with CBA, have the same spectrum as the HM (eventually with twisted boundary conditions). Here we will present the connection between DES and correlated hopping, providing a more systematic access to both types of modification.

\section{Deformed Statistics}

We define particles with DES by creation and annihilation operators obeying the following deformed commutation rules:

$$
\begin{aligned}
& f_{j, \sigma}^{\dagger} f_{k, \sigma^{\prime}}+\mathcal{Q}_{j, k}^{\sigma, \sigma^{\prime}} f_{k, \sigma^{\prime}} f_{j, \sigma}^{\dagger}=\delta_{j, k} \delta_{\sigma \sigma^{\prime}} \\
& f_{j, \sigma} f_{k, \sigma^{\prime}}+\mathcal{Q}_{k, j}^{\sigma^{\prime}, \sigma} f_{k, \sigma^{\prime}} f_{j, \sigma}=0
\end{aligned}
$$

proc.SSPCM2000.osterloh: submitted to World Scientific on August 28, 2001 
This algebra is consistent for $\mathcal{Q}_{j, k}^{\sigma^{\prime}, \sigma} \in \mathbf{C}$ if $\mathcal{Q}_{j, k}^{\sigma \sigma^{\prime}}=\left(\mathcal{Q}_{k, j}^{\sigma^{\prime}, \sigma}\right)^{-1}=\left(\mathcal{Q}_{k, j}^{\sigma^{\prime}, \sigma}\right)^{*}$. The operators $\nu_{j, \sigma} \doteq f_{j, \sigma}^{\dagger} f_{j, \sigma}$ are the particle-number operators. Note that the relation (1) is formally analog to quon commutation rules, for which however relation (2) cannot apply except for the fermionic and bosonic case ${ }^{4}$. Here, in contrast to the quon algebra, the deformation parameter depends on site and spin indices $\left(j, \sigma \mid k, \sigma^{\prime}\right)$ and there is a well defined Fock representation. ${ }^{2,5}$

The key observation is that DES as (1), (2) are realizable by means of fermionic creation/annihilation operators (termed fermionization here):

$$
f_{i, \sigma}^{\dagger}:=c_{i, \sigma}^{\dagger} \exp i\left[\Delta_{i ; l}^{\sigma ; \lambda} n_{l, \lambda}+\Delta_{i ; l, m}^{\sigma ; \lambda, \mu} n_{l, \lambda} n_{m, \mu}+\ldots\right],
$$

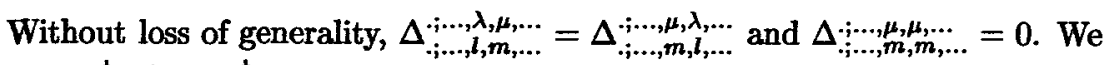
have $f_{i, \sigma}^{\dagger} f_{i, \sigma}=c_{i, \sigma}^{\dagger} c_{i, \sigma}$ and the resulting deformation parameters are

$$
\mathcal{Q}_{j, k}^{\sigma, \sigma^{\prime}}=\exp i\left[\left(\Delta_{k ; j}^{\sigma^{\prime} ; \sigma}-\Delta_{j ; k}^{\sigma ; \sigma^{\prime}}\right)+2\left(\Delta_{k ; j, m}^{\sigma^{\prime} ; \sigma, \mu}-\Delta_{j ; k, m}^{\sigma ; \sigma^{\prime}, \mu}\right) n_{m, \mu}+\ldots\right] .
$$

We see that no deformation occurs if $\Delta$ is fully symmetric in the index pairs. ${ }^{a}$ Thus, it will be assumed being antisymmetric in exchanging one index-pair behind the semicolon with the index pair in front of it. This already implies that $\Delta$ vanishes if it has more than two index pairs. Therefore

$$
\mathcal{Q}_{j, k}^{\sigma, \sigma^{\prime}}=\exp 2 \mathrm{i} \Delta_{k ; j}^{\sigma^{\prime} ; \sigma}=\exp -2 \mathrm{i} \Delta_{j ; k}^{\sigma ; \sigma^{\prime}}
$$

As a consequence it is impossible to reach correlated DES (i.e. with $\mathcal{Q}_{j, k}^{\sigma, \sigma^{\prime}}$ being a functional of $n_{m, \mu}$ ) through a realization as is (3). ${ }^{5}$

We will now focus on the Hamiltonian of the deformed HM

$$
H=-t \sum_{i, \sigma}\left(f_{i, \sigma}^{\dagger} f_{i+1, \sigma}+\text { h.c. }\right)+U \sum_{i} \nu_{i, \uparrow} \nu_{i, \downarrow},
$$

where $f_{i, \sigma}, f_{i, \sigma}^{\dagger}, \sigma \in\{\uparrow, \downarrow\}$, obey the deformed relations (1) and (2). The two contributions in the Hamiltonian are the hopping term (the $t$-term) and the Coulomb interaction term (the $U$-term). Substituting the operators $f_{i, \sigma}^{\dagger}$ by their fermionic realization, the interaction term is unaltered but the hopping term finally becomes

$$
f_{j+1, \sigma}^{\dagger} f_{j, \sigma}=c_{j+1, \sigma}^{\dagger} c_{j, \sigma} \exp i\left[\left(\tilde{\Delta}_{j+1 ; m}^{\sigma ; \mu}-\tilde{\Delta}_{j ; m}^{\sigma ; \mu}\right) n_{m, \mu}\right],
$$

where $\tilde{\Delta}$ is the same as $\Delta$ except that $\tilde{\Delta}_{j+1, j}^{\sigma ; \sigma}=0 .^{6}$

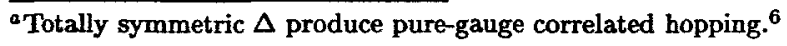

proc.SSPCM2000.osterloh: submitted to World Scientific on August 28, 20012 
Expressing the $t$-term (7) in a different notation, ${ }^{6,7}$

$$
\sum_{j, \sigma}\left\{c_{j+1, \sigma}^{\dagger} c_{j, \sigma} \exp \left(\mathrm{i} \gamma_{j}(\sigma)\right) \exp \left[\mathrm{i} \sum_{l}\left(\alpha_{j, l}(\sigma) n_{l,-\sigma}+A_{j, l}(\sigma) n_{l, \sigma}\right)\right]+\text { h.c. }\right\} \text {, }
$$

the new parameters are: $\gamma_{j}(\sigma)=0, \alpha_{j, m}(\sigma)=\tilde{\Delta}_{j+1 ; m}^{\sigma ;-\sigma}-\tilde{\Delta}_{j ; m}^{\sigma ;-\sigma}$, and $A_{j, m}(\sigma)=$ $\tilde{\Delta}_{j+1 ; m}^{\sigma ; \sigma}-\tilde{\Delta}_{j ; m}^{\sigma ; \sigma}$. Correlated hopping of type (8) is treated the next section.

\section{Correlated Hopping}

In a recent paper, H. J. Schulz and B. S. Shastry found a new class of solvable one-dimensional Hubbard and XXZ type models7. The hopping terms they studied are special cases of (8), which we were led to fermionizing DES. The aim of their approach is to unitarily map the model onto the original Hubbard or XXZ model up. to boundary twists, not affecting exact solvability: ${ }^{8}$ the Hubbard model with boundary phases $\Phi_{\sigma}$ is solvable by the Bethe ansatz $\psi(\vec{x} ; \vec{\sigma})=\sum_{\pi^{\prime}} W\left(\pi ; \pi^{\prime}\right) \exp \left(\mathrm{i} \sum_{j=1}^{W} p_{\pi^{\prime}(j)} x_{\pi(j)}\right)$. The Bethe equations are

$$
\begin{aligned}
\mathrm{e}^{\mathrm{i} p_{j} L} & =\mathrm{e}^{\mathrm{i} \Phi_{\uparrow}} \prod_{a=1}^{N_{\downarrow}} \frac{\mathrm{i}\left(\sin p_{j}-\lambda_{a}\right)-\frac{U}{4 t}}{\mathrm{i}\left(\sin p_{j}-\lambda_{a}\right)+\frac{y}{4 t}}, \\
\prod_{b=1, b \neq a}^{N_{\downarrow}} \frac{\mathrm{i}\left(\lambda_{a}-\lambda_{b}\right)+\frac{U}{3}}{\mathrm{i}\left(\lambda_{a}-\lambda_{b}\right)-\frac{U}{2 t}} & =\mathrm{e}^{\mathrm{i}\left(\Phi_{\uparrow}-\Phi_{\downarrow}\right)} \prod_{l=1}^{N} \frac{\mathrm{i}\left(\sin p_{l}-\lambda_{a}\right)-\frac{U}{4 t}}{\mathrm{i}\left(\sin p_{1}-\lambda_{a}\right)+\frac{\mathcal{U}}{4 t}} .
\end{aligned}
$$

The energy eigenvalues are given by $E=-2 t \sum_{j=1}^{N} \cos \left(p_{j}\right)$ and the eigenfunctions are obtained uniquely (up to normalization) via the $S$-Matrix. $N_{\uparrow}$ and $N_{\downarrow}$ are the total number of particles with spin up and down respectively, $L$ is the number of sites in the ring and $\lambda$ are the spin rapidities. ${ }^{9}$

We point out that the HM and the $\mathrm{XXZ}$ model with correlated hopping are not diagonalizable by direct CBA since the scattering matrix is configuration dependent. ${ }^{6}$ This can be specified in the following

Theorem 1 ( No-Go Theorem ${ }^{6}$ )

Hubbard-like models with correlated hopping

$$
c_{j+1, \sigma}^{\dagger} c_{j, \sigma} \exp \left(\mathrm{i} F_{j, \sigma}(\{n\})\right)+\text { h.c. }
$$

are solvable by direct Bethe ansatz iff $F_{j, \sigma}(\{n\})=\varphi n_{j+\Delta, \mu}$

for $\Delta \in\{0,1\}$; in case $\varphi \neq 0$ exclusively for $U=0$.

Thus, we first have to remove the phases in the hopping term up to a boundary phase, and then we apply the results of Shastry and Sutherland. ${ }^{8}$

The ansatz for the unitary transformation is

$$
\mathcal{U}:=\exp \left[\mathrm{i}\left(\xi_{l, m}^{\mu, \nu} n_{l, \mu} n_{m, \nu}+\zeta_{l, \mu} n_{l, \mu}\right)\right]=: \exp (\mathrm{i} S)_{,}
$$


(sum convention used) where $\xi_{i, j}, \zeta_{l, m} \in \mathbb{R}$ are unknown variables which have to be fixed for canceling the unitary prefactor in (8). Without loss of generality $\xi_{l, m}^{\mu, \nu}=\xi_{m, l}^{\nu, \mu}$ and $\xi_{m, m}^{\mu, \mu}=0$ (a non-zero $\xi_{m, m}^{\mu, \mu}$ can be included in the parameter $\left.\zeta_{l, m}\right)$.

We unitarily transform the Hamiltonian (6), after having inserted Eq. (7), by $\mathcal{U}: c_{j, \sigma} \stackrel{u}{\longrightarrow} U c_{j, \sigma} \mathcal{U}^{-1}$. Number operators remain unchanged, but the hopping term is altered

$$
\begin{aligned}
c_{j+1, \sigma}^{\dagger} c_{j, \sigma} \stackrel{u}{\longrightarrow} & c_{j+1, \sigma}^{\dagger} c_{j, \sigma} \exp \left[2 \mathrm{i}\left(\xi_{j+1, m}^{\sigma, \mu}-\xi_{j, m}^{\sigma, \mu}\right) n_{m, \mu}\right] \\
& \exp \left[\mathrm{i}\left(\zeta_{j+1, \sigma}-\zeta_{j, \sigma}-2 \xi_{j, j+1}^{\sigma, \sigma}\right)\right] .
\end{aligned}
$$

The unitary factors are removed if $2\left(\xi_{j, m}^{\sigma,-\sigma}-\xi_{j+1, m}^{\sigma_{1},-\sigma}\right) \stackrel{!}{=} \alpha_{j, m}(\sigma)$ for all $m$, $\left(\zeta_{j, \sigma}-\zeta_{j+1, \sigma}+2 \xi_{j, j+1}^{\sigma, \sigma}\right) \stackrel{!}{=} \gamma_{j}(\sigma)$, and $2\left(\xi_{j, m}^{\sigma, \sigma}-\xi_{j+1, m}^{\sigma, \sigma}\right) \stackrel{!}{=} A_{j, m}(\sigma)$ for $m \neq j, j+1$. Including boundary phases, these conditions are modified to be $2\left(\xi_{L, m}^{\sigma,-\sigma}-\right.$ $\left.\xi_{1, m}^{\sigma,-\sigma}\right) \stackrel{!}{=} \alpha_{L, m}(\sigma)-\phi_{\uparrow \downarrow}^{(1)}(\sigma)$ for all $m,\left(\zeta_{L, \sigma}-\zeta_{1, \sigma}+2 \xi_{L, 1}^{\sigma, \sigma}\right) \stackrel{!}{=} \gamma_{L}(\sigma)-\phi(\sigma)$, and $2\left(\xi_{L, m}^{\sigma, \sigma}-\xi_{1, m}^{\sigma, \sigma}\right) \stackrel{!}{=} A_{L, m}(\sigma)-\phi_{\uparrow}^{(1)}(\sigma)$ for $m \neq 1, L$. They constitute a system of recursive relations 'which cannot be solved for arbitrary $\alpha$ and $A$. It is solvable iff

$$
\begin{aligned}
\alpha_{j, m+1}(\sigma)-\alpha_{j, m}(\sigma) & =\alpha_{m, j+1}(-\sigma)-\alpha_{m, j}(-\sigma), \\
A_{j, m+1}(\sigma)-A_{j, m}(\sigma) & =A_{m, j+1}(\sigma)-A_{m, j}(\sigma) \text { for } m \neq j, j \pm 1 .
\end{aligned}
$$

If these "closedness conditions" are fulfilled", the correlations from the hopping term can be removed and the rotated model is finally known to be solvable by CBA $^{8}$ For open boundary conditions the correlated hopping can be "gauged away" completely, yielding the HM. Periodic boundary conditions lead to a twisted HM, whose boundary twists are given by

$$
\Phi_{\sigma}:=\phi(\sigma)+\phi_{\uparrow \downarrow}^{(1)}(\sigma) N_{-\sigma}+\phi_{\uparrow \uparrow}^{(1)}(\sigma)\left(N_{\sigma}-1\right),
$$

where the partial phases therein are obtained from the recursive relations as

$$
\begin{gathered}
\phi(\sigma)=\sum_{j=1}^{L}\left(\gamma_{j}(\sigma)+A_{j, j}(\sigma)\right) ; \quad \phi_{\uparrow \downarrow}^{(1)}(\sigma)=\sum_{j=1}^{L} \alpha_{j, m}(\sigma), \\
\phi_{\uparrow \uparrow}^{(1)}(\sigma)=\sum_{j=1, j \neq m-1, m}^{L} A_{j, m}(\sigma)+A_{m, m-1}(\sigma)+A_{m-1, m+1}(\sigma) .
\end{gathered}
$$

Due to the closedness conditions, the $m$-dependence in the above equations is only apparent.

Now we can obtain directly the relations for DES (1), (2) via Eq. (7)

$$
\operatorname{expi} \Phi_{\sigma}=\left[\mathcal{Q}_{m-1, m+1}^{\sigma, \sigma} \mathcal{Q}_{m+1, m}^{\sigma, \sigma} \mathcal{Q}_{m, m-1}^{\sigma, \sigma}\right]^{N_{\sigma}-1}
$$

${ }^{b}$ In fact modulo $2 \pi$ only. But for $A_{j, m, \ldots}^{\sigma}, \mu, \ldots, \tilde{A}_{j, m, \ldots}^{\sigma, \mu, \ldots)}$ ), zero is the only possibility. 
where $\mathcal{Q}_{j, k}^{\sigma, \sigma^{\prime}}$ has to fulfill the closedness conditions

$$
\mathcal{Q}_{j, k+1}^{\sigma, \sigma^{\prime}} \mathcal{Q}_{j+1, k}^{\sigma, \sigma^{\prime}}=\mathcal{Q}_{k, j}^{\sigma^{\prime}, \sigma} \mathcal{Q}_{k+1, j+1}^{\sigma^{\prime}, \sigma} ; \text { for } k \neq j, j \pm 1 \vee \sigma \neq \sigma^{\prime}
$$

As a consequence, for translational invariant deformations, i.e. $\mathcal{Q}_{j, k}^{\sigma, \sigma^{\prime}}=\mathcal{Q}_{k-j}^{\sigma, \sigma^{\prime}}$, the boundary phases are zero. ${ }^{5}$

To conclude, we have provided a classification of Hubbard-type models including correlated hopping as well as deformed statistics. We obtained a simple set of solvability conditions together with the corresponding Bethe equations. Only non-translational invariant deformations for particles with the same spin orientation modify the spectrum.

This work was supported by the Graduiertenkolleg "Nonlinear Problems in Analysis, Geometry, and Physics" (GRK 283), financed by the German Science Foundation (DFG) and the State of Bavaria, the SFB 484, and the INFM. Interesting and fruitful discussions with D. Braak, M. Dzierzawa, G. Falci, R. Fario, G. Giaquinta, A. Kundu, M. Rasetti, B.S. Shastry, and P. Schwab are kindly acknowledged.

\section{References}

1. A. P. Polychronakos, Generalized statistics in one dimension Topological Aspects Of Low Dimensional Systems (EDP, Springer, 1998).

2. L. Amico, A. Osterloh, and U. Eckern, Phys. Rev. B 58 (1998) 1703R; A. Osterloh, L. Amico, and U. Eckern, J. Phys. A 33 (2000) L87.

3. B. Sutherland, in Exactly Solvable Problems in Condensed Matter and Relativistic Field Theory, Lecture Notes in Physics 242 eds. B. S. Shastry, S. S. Jha, and V. Singh, (Springer Berlin, 1985); H. Bethe, Z. Phys. 71 (1931) 205.

4. O. W. Greenberg, Phys. Rev. Lett. 64, 705 (1990); Phys. Rev. D 43, 4111 (1991); L.-A. Wu, Z.-Y. Wu, and J. Sun, Phys. Lett. A 170, 280 (1992).

5. A. Osterloh, L. Amico, and U. Eckern, cond-mat/0007081 submitted to J. Phys. A.

6. A. Osterloh, L. Amico, and U. Eckern, Nucl. Phys. B 588, 531 (2000).

7. H. J. Schulz and B. S. Shastry, Phys. Rev. Lett. 80 (1998) 1924.

8. B. S. Shastry and B. Sutherland, Phys. Rev. Lett. 65 (1990) 243.

9. E. H. Lieb and F. Y. Wu, Phys. Rev. Lett. 20 (1968) 1445.

proc.SSPCM2000.osterloh: submitted to World Scientific on A ugust 28, 2001 p 